\title{
A Study of Morphological Changes in Renal Afferent Arterioles Induced by Angiotensin II Type 1 Receptor Blockers in Hypertensive Patients
}

\author{
Yohko Nagai ${ }^{a}$ f $\quad$ Fumito Yamabe $^{b}$ Yosuke Sasaki ${ }^{a} \quad$ Takamasa Ishii $^{a}$ \\ Kazushige Nakanishi ${ }^{a} \quad K$ Koichi Nakajima ${ }^{b}$ Kazutoshi Shibuya ${ }^{c}$ \\ Tetsuo Mikamid ${ }^{d}$ Yoshikiyo Akasaka ${ }^{e}$ Yoshihisa Urita ${ }^{a}$ \\ Nobuaki Yamanaka ${ }^{f}$ \\ ${ }^{a}$ Department of General Medicine and Emergency Care, Toho University, Tokyo, Japan; \\ ${ }^{b}$ Department of Urology, Toho University, Tokyo, Japan; ' Department of Surgical Pathology, \\ Toho University, Tokyo, Japan; ${ }^{d}$ Department of Pathology, Toho University, Tokyo, Japan; \\ e Center of Advanced Research, Toho University, Tokyo, Japan; ${ }^{\mathrm{f}}$ Tokyo Kidney Research \\ Institute, Tokyo, Japan
}

\section{Keywords}

Angiotensin II receptor blockers $\cdot$ Renal arteriole $\cdot$ Smooth muscle cells $\cdot$ Renin-angiotensinaldosterone system $\cdot$ Hypertension

\begin{abstract}
Background: Renin-angiotensin-aldosterone system blockers are known to reduce hypertrophy of vascular smooth muscle cells (SMCs) in hypertensive cases. However, we have reported marked proliferative changes of renal afferent arteriolar SMCs in rats induced by a long-term administration of angiotensin II type 1 receptor blockers (ARBs) and an angiotensin-converting enzyme inhibitor (ACEI). In this study, we examined the morphological changes of afferent arteriolar walls in human kidneys with or without ARBs/ACEls. Methods: Forty-four wedge resections were taken from patients aged 45-74 years from 92 nephrectomized kidneys due to malignancy at Toho University Omori Medical Center between 2013 and 2016. They were divided into the following three groups: 18 hypertensive patients treated with antihypertensive agents including ARBs or ACEls (the HTARB group), 6 hypertensive patients treated with calcium channel blockers without ARBs/ACEls (the HTCCB group), and 20 normotensive patients (the normotensive group) as a control. Cases expecting vascular changes such as diabetes were excluded. In each case renal arterioles were measured as the ratio of inner/outer arteriolar diameter, and pathologists estimated morphological abnormal changes, scor-
\end{abstract}




\section{Kidney \\ Blood Pressure \\ Research}

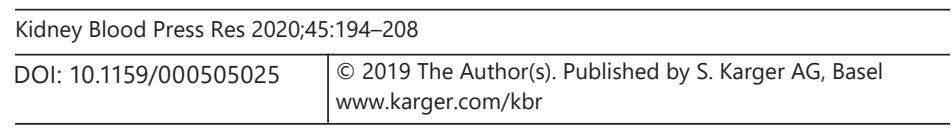

Nagai et al.: Morphological Changes of Renal Arterioles Induced by ARB Treatment

ing each specimen independently. Results: The ratio in the HTARB group was $0.39 \pm 0.05$ (mean $\pm \mathrm{SD}$ ), and was significantly the lowest among the three groups $(0.46 \pm 0.02$ in the HTCCB, $0.53 \pm 0.02$ in the normotensive group; $p=0.0107$ vs. HTCCB, $p=0.00001$ vs. normotensive). The ratio in the three groups significantly correlated with the estimated glomerular filtration rate $(r=0.4915, p<0.0007)$. The afferent arteriolar SMCs in the HTARB group frequently showed marked proliferative and irregular changes. The score of SMC abnormalities estimated regarding the proliferation, irregularity of the arrangement, and size in hilar afferent arteriolar SMCs was highest in the HTARB group and showed statistical significance ( $p=$ $0.0088, p=0.00001$, and $p=0.025$ versus other two groups). Conclusions: We consider that these morphological changes in arterioles are induced by ARBs/ACEls. These changes could induce an important suppression of glomerular hyperfiltration and could lead to glomerular ischemia. However, the clinical consequences of these morphological changes in correlation with ARBs/ACEls were not sufficiently clear and require further analysis. We should consider renal arteriolar morphological changes when using ARBs/ACEls. (c) 2020 The Author(s)

\section{Introduction}

We have reported in several previous publications that the long-term administration of angiotensin II type 1 receptor blockers (ARBs) [1,2] and angiotensin-converting enzyme inhibitors (ACEIs) [3] induced marked proliferative changes in renal afferent arteriolar walls. We found those morphological changes of arteriolar walls in the kidneys of some varied strains of rats. These days, among antihypertensive agents, ARBs/ACEIs are highly important due to their cardioprotective and renoprotective effects $[4,5]$. Therefore, we investigated whether the same morphological changes to afferent arterioles would appear in human kidneys.

\section{Methods}

\section{Subjects}

Ninety-two wedge resections in uninvolved parts of kidneys with malignant neoplasm were performed from uninephrectomized kidneys in the Department of Urology, Toho University, Omori Medical Center from 2013 to 2016. The majority of the kidneys were uninephrectomized because of renal cell carcinoma or urothelial carcinoma. Among all of the cases, patients who we expected to have pre-existing vascular abnormalities, such as 18 cases with diabetes mellitus, 2 cases with atrophic kidney because of staghorn calculus and chronic pyelonephritis, 1 case of end-stage kidney disease with hemodialysis, and 1 case with progressive systemic sclerosis, were excluded from this study. Elderly patients over 75 years old were also excluded, according to the definition and classification of aged people according to the Japan Gerontological Society, determined in January 2017: pre-old (65-74 years), old (75-89 years), super-old (90+ years) [6]. Because the number of cases aged over 75 years was relatively small and, moreover, vascular changes associated with aging naturally might include modified renal arteriolar morphological features, we considered it to be difficult to estimate the morphological abnormalities induced by antihypertensive agents correctly in those cases aged between 75 and 94 years. As all of the hypertensive patients were relatively older than the normotensive patients, we also excluded patients aged younger than their early 40 s for the age-matched study.

Finally, 44 specimens were divided into three groups and were studied. Eighteen hypertensive patients were treated with antihypertensive agents including ARBs or ACEIs (the 


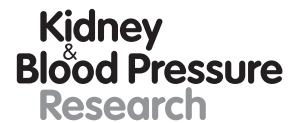

Kidney
Blood Pressure
Research

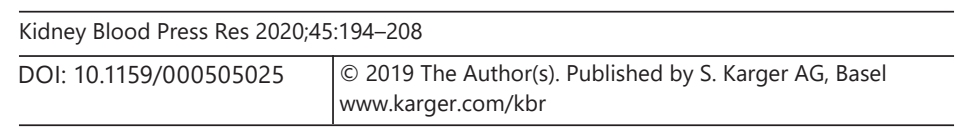

Nagai et al.: Morphological Changes of Renal Arterioles Induced by ARB Treatment

HTARB group: candesartan, $n=4$; valsartan, $n=4$; telmisartan, $n=3$; olmesartan, $n=3$; irbesartan, $n=2$; losartan, $n=1$, and perindopril, $n=1$ ). In the HTARB group, 3 cases were treated with ARBs alone, 9 cases with ARBs and calcium channel blockers (CCBs), 2 cases with ARBs and diuretics, 2 cases with ARBs, CCBs, and diuretics, 1 case with ARBs and $\alpha$-blockers, and 1 case with ACEIs and CCBs, respectively. Six hypertensive patients were treated with CCBs without renin-angiotensin system (RAS) blockers (the HTCCB group: amlodipine, $n=5$, and cilnidipine, $n=1$ ). Twenty patients were normotensive and studied as the control group.

\section{Procedures}

We compared the clinical features of these three groups, including height, body weight, body mass index (BMI), and blood pressure on admission. Serum creatinine levels and the estimated glomerular filtration rate (eGFR), total cholesterol levels, triglycerides, and urinalysis before nephrectomy were also studied. The existence of a cardiomegaly and calcifications of the aortic arch on X-ray and electrical left ventricular hypertrophy on electrocardiogram were studied before the operation in each patient. An abdominal CT scan was performed in each patient. These clinical features were then statistically analyzed.

\section{Histological Analysis}

Wedge resections were performed just after uninephrectomies and were used for light microscopic studies and immunohistochemistry. A part of them was used for electron microscopic studies. Specimens for light microscopic examination and immunohistochemistry were fixed in 10\% neutral-buffered formalin solution and embedded in paraffin. Blocks for microscopic examination from each of the three groups were sectioned at $2 \mu \mathrm{m}$ and stained with hematoxylin and eosin (HE), periodic acid-silver methenamine-HE (PASM-HE), and Elastica-Masson-Trichrome (E-MT) stain. The sections were examined by 2 pathologists independently.

Glomerular numbers were counted and the percentage of global sclerosis in all glomeruli was calculated in each specimen. We counted glomerular numbers under 100 -fold magnification with a light microscope using a manual counter. We captured the stained whole section and measured the cortex area of each specimen using Photoshop CC (Adobe, San Jose, CA, USA). We took 40-50 microphotographs (Olympus BX61; Olympus Co., Tokyo, Japan) of each sample in PASM-HE by 200 -fold magnification. We considered arteriolar walls with one or more layers of smooth muscle cells (SMCs) which continued to the beginning of Bowman's capsule in the glomerular hilus as afferent arterioles. The normal SMCs of afferent arteriolar walls show cuboidal shapes of almost the same size, regularly arranged like cobble stones along the arteriolar lumen. We considered arterioles with one flat layer of SMC wall as efferent arterioles. The efferent arterioles branch to the peritubular capillary plexus soon after leaving the glomerular hilus. Then, we considered arterioles with SMC walls apart from glomeruli, except interlobular arteries, as afferent arterioles. We selected vertical sections of afferent arterioles with an outer dimeter of 20-70 $\mu \mathrm{m}$. The outer diameter of afferent arterioles was considered as the outermost layer of arteriolar SMC layers to the same point on the opposite side. The inner diameter of afferent arterioles was measured from the innermost layer of SMCs in the same way as above. In afferent arterioles, the endothelial cell line is very thin, so we avoided the point of the nuclei of endothelial cells when we made the measurement. We measured the outer and inner diameter by manual measurement in microphotographs with 200 -fold magnification using Photoshop CC. We calculated the ratio of inner/outer diameter in each arteriole and summed them up in each case, and averaged the mean values in the three groups, respectively. We analyzed the data statistically among the three groups.

In the microphotographs, glomeruli accompanied with hilus and afferent arteriole (sometimes both of afferent and efferent), were also estimated regarding the proliferation and 


\section{Kidney \\ Blood Pressure \\ Research}

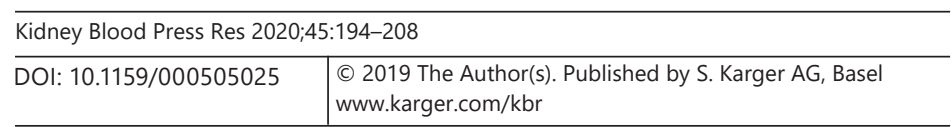

Nagai et al.: Morphological Changes of Renal Arterioles Induced by ARB Treatment

irregularity of the arrangement or size in afferent arteriolar SMCs. We scored the increased numbers of SMC nuclei in the arteriolar wall as proliferative change (marked as $2+$ ), moderate $(1+)$, and within the normal limit (0). With irregularity in the size of SMCs scored $2+$ the biggest one was more than three times compared to the smallest. In the moderate $1+$ band, the biggest SMC was twice as big as the smallest. The irregularities of SMC arrangements in the arteriolar walls were scored as: irregular, $1+$, and within normal limits, 0 . We calculated the mean scores in each case and their data were also analyzed statistically among the three groups.

The numbers of nuclei in the afferent arteriolar SMCs were counted in microphotographs with 200-fold magnification using a manual counter. We picked up and counted arterioles completely vertically sectioned without the glomerular hilus in each case, totaling around 50 arterioles in each group.

We studied and counted interlobular, arcuate, and interlobar arteries shown in each section stained by E-MT stain in each group. We measured the outer and inner diameter of each artery. We also measured the thickness of the muscular layer and the elastofibrotic layer with Elastica staining in each artery with microphotographs of 100- or 200-fold magnification using Photoshop CC. We studied the arteries completely vertically or horizontally sectioned.

The interstitial fibrotic areas in each specimen were measured with E-MT stain using Photoshop CC. We took 13 microphotographs of the superficial cortex along the renal capsule and 13 microphotographs of the deep cortex with an Olympus BX61 (Olympus Co.), which did not overlap, with low magnification (50-fold). We summed up the measured data of the fibrotic area of the superficial cortex and deep cortex differently. We calculated the percentages of the occupying fibrotic area to total area in the superficial cortex and the deep cortex in each group, and performed statistical comparisons among the three groups.

\section{Immunohistochemistry}

The paraffin blocks mentioned above were also used for immunohistochemistry. The sections underwent deparaffinization and rehydration. The primary antibody used was polyclonal anti-human renin sheep antibody (R\&D systems, Minneapolis, MN, USA) and the secondary antibody used was polyclonal rabbit anti-sheep immunoglobulins/HSP (Dako, Carpinteria, CA, USA). The other primary antibodies were monoclonal anti-human $\alpha$-smooth muscle actin antibody (SMA; Dako) and monoclonal anti-human smooth muscle myosin heavy chain isoform 1 and 2 antibodies (SM-1, SM-2; Yamasa, Tokyo, Japan). The secondary antibody except renin used was LSAB2 system/HRP (Dako). The positive renin expression in the glomerular hilus was calculated as the percentage of total numbers of glomeruli excluding global sclerotic glomeruli in each case, and were statistically analyzed in the three groups.

SM-1 and SM-2 were treated by microwave for $90 \mathrm{~min}$ in buffer ( $\mathrm{pH} 10$ ) using the antigen retrieval technique. To verify antibody specificity, sections from each paraffin block were used as negative controls by omitting the primary antibodies. Specimens were observed using an Olympus BX63 (Olympus Co.). We examined the afferent arteriole which had been serially stained with anti-SMA, SM-1 and SM-2 monoclonal antibodies in the one arteriole. We estimated and compared the proportion of the three antibodies in the three groups.

\section{Electron Microscopic Studies}

The specimens for electron microscopy were fixed in 5\% glutaraldehyde solution, postfixed in osmium, embedded in Epon resin, sliced into ultrathin sections, and then examined under an electron microscope (JEM-1400; JOEL, Tokyo, Japan). Tannic acid staining was performed using Oolong tea extract solution (Suntory Holdings Ltd., Osaka, Japan). 
Table 1. Clinical features of the three groups

\begin{tabular}{lcccc}
\hline & HTARB group & HTCCB group & $\begin{array}{l}\text { Normotensive } \\
\text { group }\end{array}$ & $p$ value \\
& & & 20 & \\
$n$ & 18 & 6 & 65 & 0.3145 \\
Male, \% & 50 & 83.3 & $60.7 \pm 7.8$ & $0.8637^{\mathrm{a}}, 0.1692^{\mathrm{b}}, 0.0833^{\mathrm{c}}$ \\
Age at nephrectomy, years & $64.4 \pm 8.1$ & $67.3 \pm 3.3$ & $22.4 \pm 4.3$ & $0.7918^{\mathrm{a}}, 0.1223^{\mathrm{b}}, 0.7523^{\mathrm{c}}$ \\
BMI & $25.1 \pm 6.3$ & $23.0 \pm 2.3$ & $127.7 \pm 14.9$ & $0.3079^{\mathrm{a}}, 0.3782^{\mathrm{b}}, 0.0838^{\mathrm{c}}$ \\
Systolic BP on admission & $133.4 \pm 13.4$ & $143.8 \pm 16.5$ & $79.0 \pm 10.8$ & $0.2890^{\mathrm{a}}, 0.5545^{\mathrm{b}}, 0.1251^{\mathrm{c}}$ \\
Diastolic BP on admission & $82.0 \pm 10.1$ & $88.6 \pm 10.1$ & $62.2 \pm 14.5$ & $0.9896^{\mathrm{a}}, 0.295^{\mathrm{b}}, 0.5780^{\mathrm{c}}$ \\
scCr, $\mu$ mol/L & $74.2 \pm 22.2$ & $68.7 \pm 9.7$ & $84.5 \pm 20.9$ & $0.2927^{\mathrm{a}}, \mathbf{0 . 0 0 9 9}^{\mathrm{b}}, 0.4090^{\mathrm{c}}$ \\
eGFR, mL/min/1.73m ${ }^{2}$ & $64.4 \pm 14.1$ & $73.8 \pm 8.9$ & $198.9 \pm 57.6$ & $0.5298^{\mathrm{a}}, 0.9127^{\mathrm{b}}, 0.8560^{\mathrm{c}}$ \\
Total cholesterol & $187.6 \pm 31.0$ & $202.7 \pm 25.1$ & 30 & 0.4315 \\
Hyperlipidemia, \% & 50 & 33.3 & 15 & 0.6029 \\
Cardiomegaly on chest X-ray, \% & 27.8 & 16.7 & 15 & 0.2080 \\
Calcification of aortic arch, \% & 38.9 & 16.7 & 15 & 0.6029 \\
LVH by voltage criteria on EKG, \% & 27.8 & 16.7 & 75 & 0.1769 \\
Smoking & 38.9 & 66.7 & 15 & 0.1913 \\
Macrohematuria, \% & 38.9 & 0 & 0.1539 \\
Hydronephrosis, \% & 16.7 & 50 & & \\
\hline
\end{tabular}

Values are presented as the mean \pm SD. The significant $p$ value is in bold. BP, blood pressure; s-Cr, serum creatinine; eGFR, effective glomerular filtration rate; LVH, left ventricular hypertrophy.

${ }^{a}$ HTARB group versus HTCCB group. ${ }^{b}$ HTARB group versus normotensive group. ${ }^{c}$ HTCCB group versus normotensive group.

\section{Statistical Analysis}

Values are presented as the mean \pm standard deviation. The Kruskal-Wallis test was performed to assess the significance of results followed by the Steel-Dwass test for multiple comparisons. Differences in proportion were tested by the $\chi^{2}$ test. Values of $p<0.05$ were considered to be significant.

\section{Results}

\section{Clinical Features}

The clinical features of the three groups are shown in Table 1. The age, BMI, blood pressure on admission, and serum creatinine levels before operation showed no significant differences among the three groups. The mean eGFRs in the three groups were 64.4 $\pm 14.0 \mathrm{~mL} / \mathrm{min} / 1.73 \mathrm{~m}^{2}$ in the HTARB group, $73.8 \pm 8.9 \mathrm{~mL} / \mathrm{min} / 1.73 \mathrm{~m}^{2}$ in the HTCCB group, and $84.5 \pm 20.9 \mathrm{~mL} / \mathrm{min} / 1.73 \mathrm{~m}^{2}$ in the normotensive group. The eGFR was significantly lower in the HTARB group compared to the normotensive group $(p<0.001)$. Averaged total cholesterol levels in the three groups were not significantly different. The numbers of patients with hyperlipidemia were 9 out of 18 cases in the HTARB group, 2 out of 6 cases in the HTCCB group, and 6 out of 20 cases in the normotensive group. Almost all hyperlipidemia patients were treated with statins, with the exception of 7 cases. The frequency of cardiomegaly and the calcification of thoracic aorta in chest X-ray showed no statistical significance among the three groups. Left ventricular hypertrophy by voltage criteria on electrocardiogram also showed no significant differences among the three groups. The cases of hydronephrosis detected by CT images in the three groups were not significantly different. 
Table 2. Histological findings of the three groups

\begin{tabular}{|c|c|c|c|c|c|c|}
\hline & \multirow{2}{*}{$\begin{array}{l}\text { HTARB } \\
\text { group }\end{array}$} & \multirow{2}{*}{$\begin{array}{l}\text { HTCCB } \\
\text { group }\end{array}$} & \multirow{2}{*}{$\begin{array}{l}\text { Normotensive } \\
\text { group }\end{array}$} & \multicolumn{3}{|l|}{$p$ value } \\
\hline & & & & $\begin{array}{l}\text { ARB vs. } \\
\text { CCB }\end{array}$ & $\begin{array}{l}\text { ARB vs. } \\
\text { normo. }\end{array}$ & $\begin{array}{l}\text { CCB vs. } \\
\text { normo. }\end{array}$ \\
\hline$n$ & 18 & 6 & 20 & & & \\
\hline Total glomeruli per specimen, $n$ & $517 \pm 223$ & $556 \pm 166$ & $446 \pm 185$ & 0.8336 & 0.6174 & 0.2679 \\
\hline Global sclerosis, $\%$ & $12.1 \pm 11.0$ & $8.5 \pm 4.2$ & $4.9 \pm 4.1$ & 0.9544 & $0.0061^{* *}$ & 0.1344 \\
\hline Measured arterioles, $n$ & 661 & 347 & 969 & & & \\
\hline Measured arterioles per case, $n$ & $39 \pm 6.6$ & $43 \pm 10.3$ & $42 \pm 7.8$ & 0.6650 & 0.2715 & 0.9927 \\
\hline Media/lumen ratio & $0.3894 \pm 0.05$ & $0.4590 \pm 0.02$ & $0.5298 \pm 0.04$ & $0.01069 *$ & $0.00001^{* *}$ & $0.0041^{* *}$ \\
\hline Glomerular hili with afferent & & & & & & \\
\hline $\begin{array}{l}\text { arterioles per case, } n \\
\text { Morphological abnormality score of }\end{array}$ & $13.1 \pm 5.1$ & $14.8 \pm 6.7$ & $14.1 \pm 4.6$ & 0.8957 & 0.7306 & 0.9804 \\
\hline $\begin{array}{l}\text { hilar afferent arterioles per case } \\
\text { Hilar afferent arterioles with }\end{array}$ & $2.47 \pm \pm 0.6$ & $1.50 \pm 0.4$ & $0.98 \pm 0.3$ & $0.0088^{* *}$ & $0.0000^{* *}$ & $0.025^{*}$ \\
\hline $\begin{array}{l}\text { morphological abnormalities, } \% \\
\text { Fibrotic area by MT stain }\end{array}$ & $83.1 \pm 12.8$ & $47.6 \pm 10.1$ & $32.2 \pm 15.8$ & $0.0016^{* *}$ & $0.00001^{* *}$ & $0.0398^{*}$ \\
\hline In superficial cortex, $\%$ & $2.6 \pm 1.3$ & $3.7 \pm 1.2$ & $2.8 \pm 2.4$ & 0.1601 & 0.9001 & 0.4325 \\
\hline In deep cortex, $\%$ & $2.5 \pm 1.1$ & $4.6 \pm 1.6$ & $3.0 \pm 3.0$ & $0.0404^{*}$ & 0.8241 & 0.0728 \\
\hline
\end{tabular}

Values are presented as the mean \pm SD. ${ }^{*} p<0.05,{ }^{* *} p<0.01$. MT stain, Masson-Trichrome stain; ARB, HTARB group; CCB, HTCCB group; normo., normotensive group.

\section{Histological Findings}

The histological findings are shown in Table 2. The numbers of glomeruli observed in the three groups were $517.3 \pm 233.1$ in the HTARB group, $556.5 \pm 166.7$ in the CCB group, and $446.2 \pm 185.4$ in the normotensive group, in each specimen, respectively. The averaged percentages of global sclerosis in all glomeruli in each group were $12.1 \pm 11.0,8.5 \pm 4.2$, and $4.9 \pm 4.2 \%$ in the three groups, respectively. The numbers of observed glomeruli showed no significant differences among the three groups. However, the percentage of global sclerotic glomeruli in the HTARB group was significantly higher than in the normotensive group $(p<$ 0.01). Almost all other glomeruli showed minor glomerular abnormalities except small numbers of glomeruli with mild mesangial expansion. The cortex area of one section used for the studies was $1.5 \pm 0.4 \mathrm{~cm}^{2}$ in the HTARB group, $2.8 \pm 3 \mathrm{~cm}^{2}$ in the HTCCB group, and $1.2 \pm$ $0.6 \mathrm{~cm}^{2}$ in the normotensive group. There were no significant differences among the three groups. The mean numbers of measured arterioles in each specimen showed no statistical difference.

The inner/outer diameter ratio of vertically sectioned afferent arterioles were averaged in each group $(0.3894 \pm 0.05$ in the HTARB group, $0.4590 \pm 0.02$ in the HTCCB group, and $0.5298 \pm 0.04$ in the normotensive group). The data in the two hypertensive groups were statistically lower than in the normotensive group ( $p<0.05, p<0.0001$, respectively). The data in the HTARB group were the lowest, and a statistically significant difference was also shown between the two hypertensive groups (HTARB group vs. HTCCB group, $p<0.01$ )

The number of glomerular hili with afferent arterioles per case could be observed as 13.1 $\pm 5.6,14.8 \pm 8.7$, and $14.1 \pm 4.5$ in each group, respectively (Table 2). The afferent arteriolar SMCs at the glomerular hilus in the HTARB group frequently showed marked proliferative changes, showing thickening of the arteriolar wall. The size and the arrangement of the SMCs in the wall were remarkably irregular. The glomeruli showed minor glomerular abnormalities (Fig. 1a). The afferent arteriolar walls in the HTCCB group sometimes showed thickening, and the numbers of SMCs increased. However, the size and the arrangement of the 


\section{Kidney \\ Blood Pressure Research}

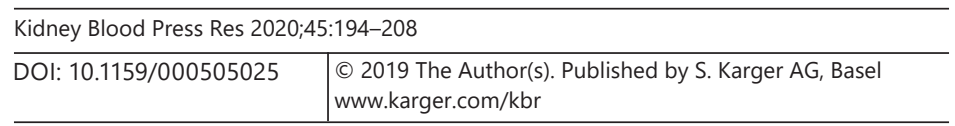

Nagai et al.: Morphological Changes of Renal Arterioles Induced by ARB Treatment
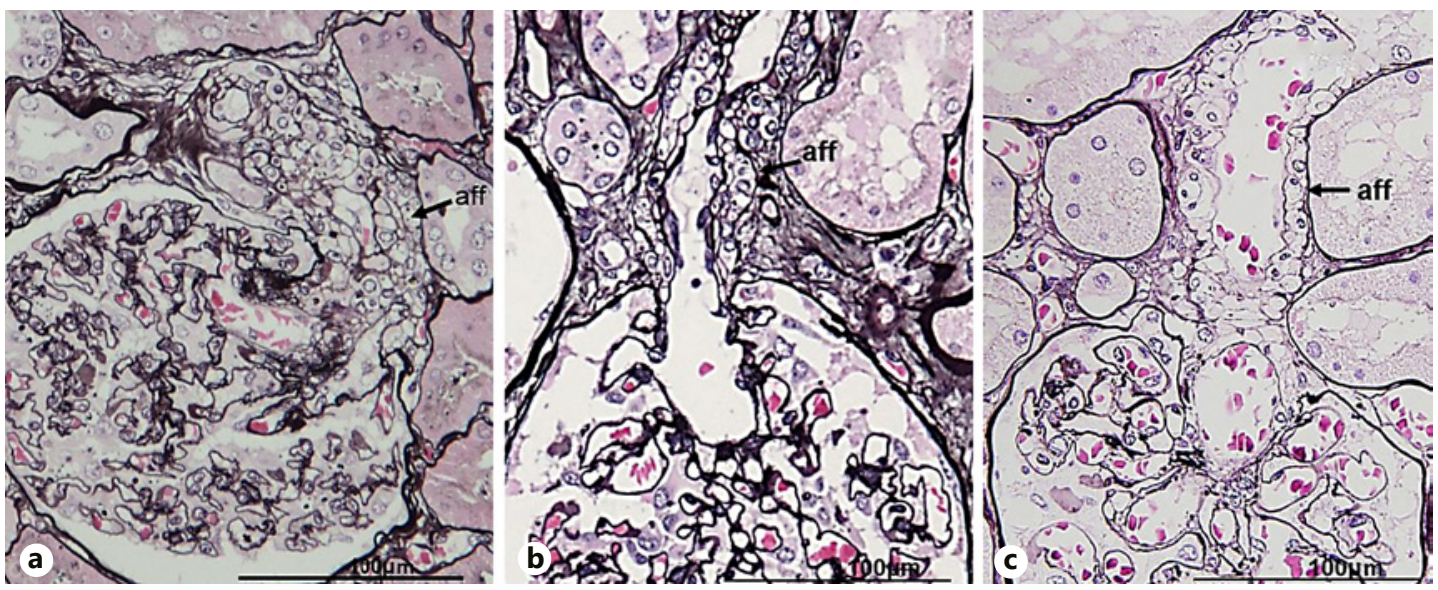

Fig. 1. Light microphotographs of the three groups. a The SMCs of afferent arteriolar walls show extreme proliferative changes, irregular arrangement, and varying size in the HTARB group. The changes in the arteriolar walls are different and more serious than those usually observed in hypertensive arteriolosclerosis. The arteriolar lumen is noticeably narrowed. $\mathbf{b}$ The afferent arteriolar walls in the HTCCB group are thicker than in the normotensive group; however, the proliferation, irregularity of arrangement, and cellular size of SMCs are milder than those of the HTARB group. c The afferent arteriolar walls always consist of one or two layers of the SMCs, showing regular arrangement and size in the normotensive group. Original magnification, $\times 200$. aff, afferent arteriole.

SMCs in the wall usually showed mild irregularities (Fig. 1b). The afferent arteriolar walls in the normotensive group showed thickening in some cases of patients aged in their $60 \mathrm{~s}$ and $70 \mathrm{~s}$, but the size and the arrangement of the SMCs in the walls showed very mild irregularities (Fig. 1c). Extremely high total scores of proliferative changes, irregular arrangement, and irregularity of the size of SMCs were observed in the HTARB group.

The numbers of nuclei in SMC walls of afferent arterioles without a glomerular hilus were measured as $25.7 \pm 6.1$ in the HTARB group $(n=54), 19.9 \pm 6.0$ in the HTCCB group $(n=48)$, and $14.3 \pm 4.8$ in the normotensive group $(n=60)$. The number of nuclei in the HTARB group was significantly higher than in the other two groups $(p<0.001)$.

The scores of morphological abnormalities in afferent arteriolar SMCs were estimated by the proliferation, irregularity of the arrangement, and size. The averaged percentage of afferent arterioles with one or more of these three abnormalities to the total observed glomeruli with hilus was significantly the highest in the HTARB group $(83.1 \pm 12.8 \%$ vs. $47.6 \pm$ $10.1 \%$ in the HTCCB group and $32.2 \pm 15.8 \%$ in the normotensive group; $p<0.01, p<0.0001$, $p<0.05$, respectively).

The percentages of interstitial fibrotic areas to all observed areas taken by microphotographs showed no statistical significance among the three groups. In the superficial cortex, only the fibrotic area in the HTCCB group in the deep cortex was statistically wider than in the HTARB group $(p<0.05)$.

In the immunohistochemical studies, the positive expression of renin was more frequently seen in the HTARB group than the HTCCB and the normotensive groups. However, statistical significance was not found among the groups $(13.5 \pm 13.8 \%$ in the ARB group, $4.0 \pm 4.6 \%$ in the CCB group, and $7.7 \pm 6.2 \%$ in the normotensive group; Fig. 2a-c). The renal afferent arteriolar walls with proliferative changes and irregularities of arrangement and size of SMCs showed a striking decrease in the positive expression of smooth muscle myosin isoforms, especially SM-2 (Fig. 3a-i). 
Kidney
Blood Pressure

Research

\begin{tabular}{l|l}
\hline Kidney Blood Press Res 2020;45:194-208 \\
\hline DOI: 10.1159/000505025 & $\begin{array}{l}\text { @ 2019 The Author(s). Published by S. Karger AG, Basel } \\
\text { www.karger.com/kbr }\end{array}$ \\
\hline
\end{tabular}

Nagai et al.: Morphological Changes of Renal Arterioles Induced by ARB Treatment
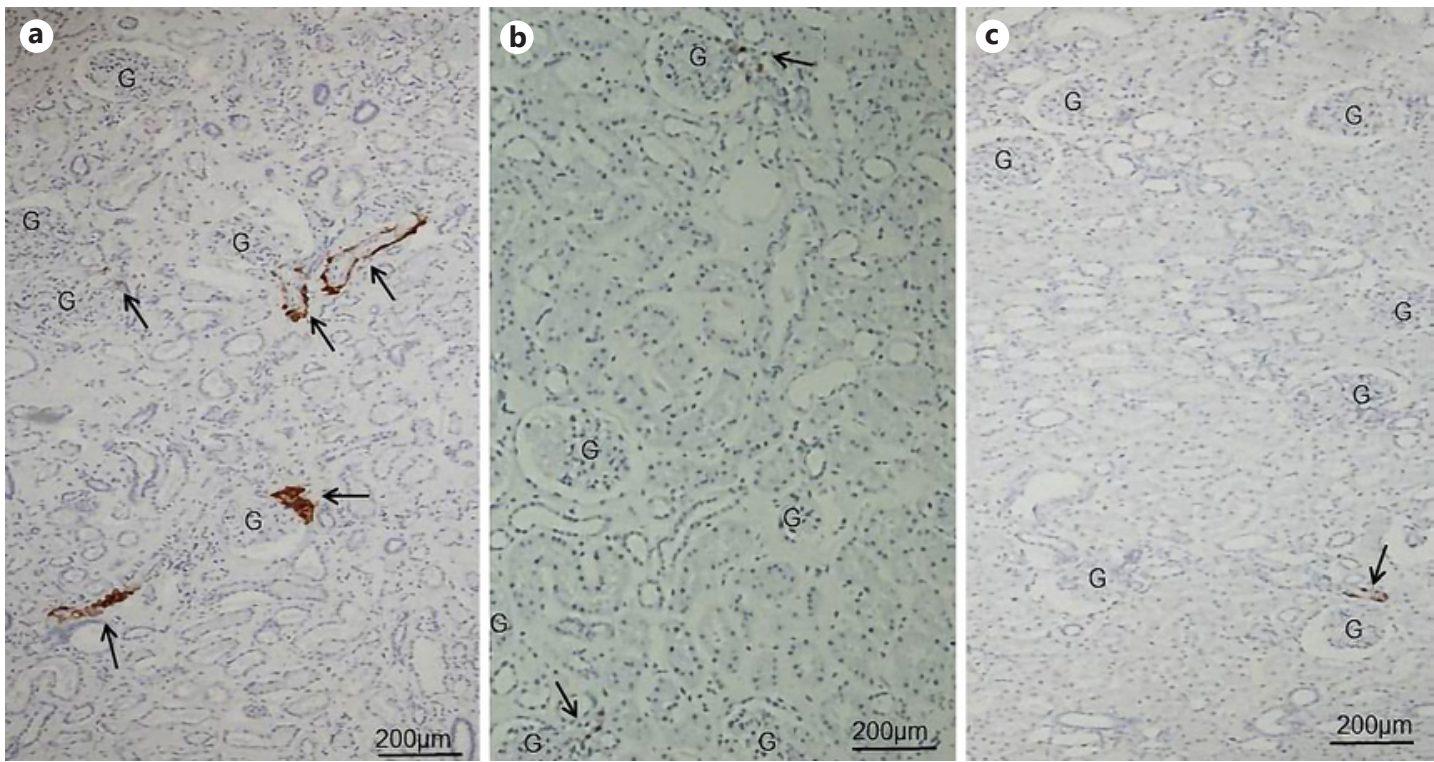

Fig. 2. The renin expression of immunohistochemical studies of the three groups. a The HTARB group shows strong positive renin expression in the glomerular hilus and arteriolar walls (arrows). The renin expression was sometimes observed clearly along the outer layer of SMCs. b The HTCCB group shows the renin expression (arrows) to be smaller and much less frequent than the HTARB group. c The normotensive group also shows the renin expression (arrow) infrequently. Original magnification, ×40. G, glomerulus.

Electron microphotographs in the HTARB group showed multilayered, unusually proliferative changes to afferent arteriolar SMC walls and narrowed arteriolar lumens. In the HTARB group, the irregularity of the arrangement and the size in the SMCs of afferent arteriolar walls was also strikingly increased (Fig. 4). A remarkable increase of renin granules was sometimes observed in the SMCs of the outer layer, especially in some patients treated with ARBs and diuretics

The relationship between all data of the ratio of the inner/outer arteriolar diameter in the three groups and eGFR are shown in Figure 5a, and the mean blood pressure is shown in Figure $5 \mathrm{~b}$. The ratio of the inner/outer arteriolar diameter in the three groups significantly correlated with eGFR $(r=0.4915, p<0.001)$ but did not correlate with the mean blood pressure.

Larger arteries in the three groups were studied. In the interlobular arteries, the outer diameter was $144.5 \pm 55.1 \mu \mathrm{m}(n=281 ; 15.6 \pm 4.6$ /specimen $)$ in the HTARB group, $137.2 \pm$ $35 \mu \mathrm{m}(n=98 ; 16.3 \pm 4.6$ /specimen) in the HTCCB group, and $147.2 \pm 37.8 \mu \mathrm{m}(n=256 ; 13.5$ \pm 6.3 /specimen) in the normotensive group. The ratio of inner/outer arteriolar diameter and thickness of vascular walls were studied. However, we found no significant differences among the three groups.

In the arcuate arteries, the outer diameter was $408.3 \pm 84.6 \mu \mathrm{m}(n=128 ; 7.1 \pm 3.5$ / specimen) in the HTARB group, $415 \pm 38 \mu \mathrm{m}(n=49 ; 8.1 \pm 3.3 /$ specimen $)$ in the HTCCB group, and $384 \pm 69.2 \mu \mathrm{m}(n=125 ; 6.4 \pm 3.5$ /specimen $)$ in the normotensive group. The percentage of the thickness of the muscular wall to the outer diameter was $23.8 \pm 6.7 \%$ in the HTARB group and $20.3 \pm 5.1 \%$ in the HTCCB group. The percentages of both groups were statistically higher than that of the normotensive group $(11.9 \pm 3.0 \%, n=128,6.4 \pm 3.5$ /specimen; $p<$ $0.0001, p<0.005$, respectively). Elastofibrosis showed no significant differences among the three groups. 


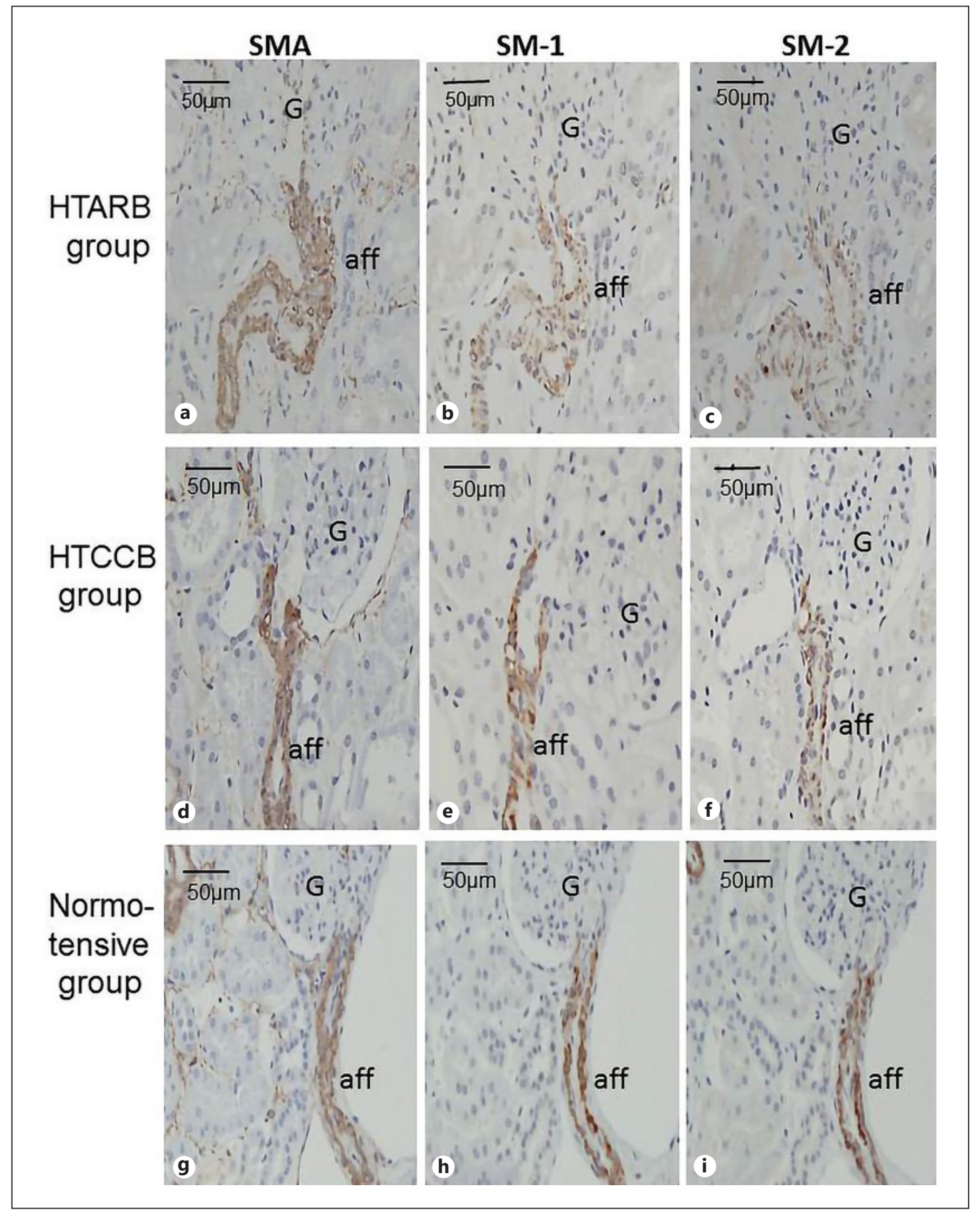

Fig. 3. Immunohistochemical studies of contractile fibers in afferent arterioles. SMA shows a clear positive expression in afferent arteriolar walls in the three groups $(\mathbf{a}, \mathbf{d}, \mathbf{g})$. The afferent arteriolar walls in the HTARB group are thick and winding. SM-1 is clearly positive in the normotensive group (h), almost plosive in the HTCCB group (e), and less positive in the HTARB group (b). SM-2 is also clearly positive in the normotensive group (i), less positive or sometimes very weak in the HTCCB group (f), and it was very weak or completely negative in the HTARB group (c). Original magnification, $\times 200$. G, glomerulus; aff, afferent arteriole. 
Kidney

Blood Pressure

Research

Fig. 4. Electron microphotograph of an arteriole in the THARB group. An electron microscopic photograph showing an arteriole with tannic acid staining. The irregularly shaped and proliferated SMCs surround the arteriolar lumen (arrow). The media is unusually thickened and the lumen is extremely narrowed for an arteriole of this size. Original magnification, $\times 2,000$. En, endothelial cell; SMC, smooth muscle cell.

\begin{tabular}{l|l}
\hline Kidney Blood Press Res 2020;45:194-208 \\
\hline DOI: 10.1159/000505025 & $\begin{array}{l}\text { @ 2019 The Author(s). Published by S. Karger AG, Basel } \\
\text { www.karger.com/kbr }\end{array}$ \\
\hline
\end{tabular}

Nagai et al.: Morphological Changes of Renal Arterioles Induced by ARB Treatment

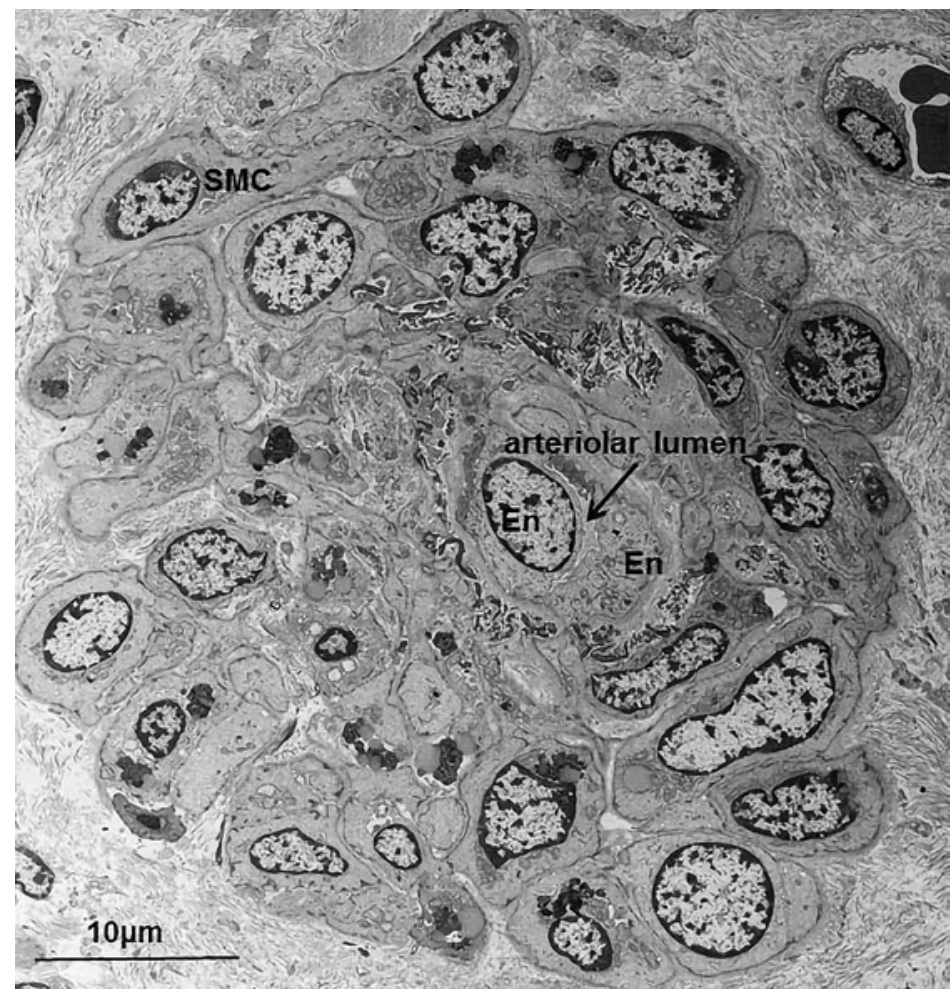

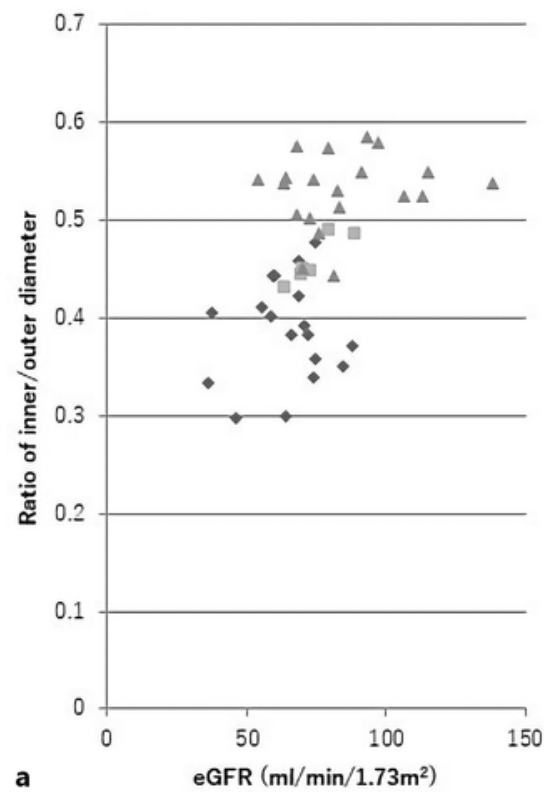

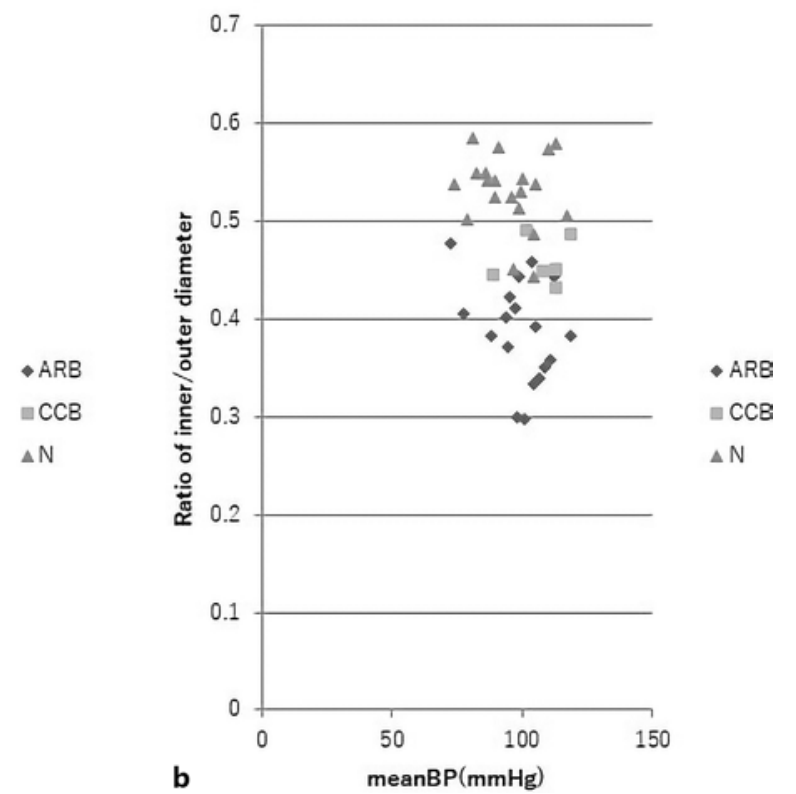

Fig. 5. Relationship between the ratio of the inner/outer diameter of arterioles and eGFR (a) and mean blood pressure (BP; b). The ratio of the inner/outer diameter of arterioles in the three groups correlated with eGFR significantly ( $r=0.4915, p<0.001)$. ARB, HTARB group; CCB, HTCCB group; N, normotensive group. 


\section{Kidney \\ Blood Pressure \\ Research}

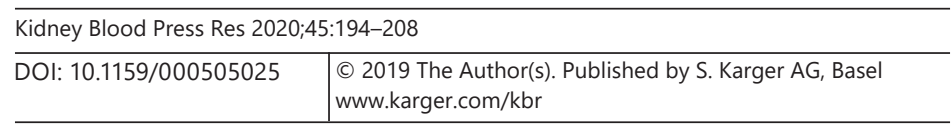

Nagai et al.: Morphological Changes of Renal Arterioles Induced by ARB Treatment

In the interlobar arteries, the outer diameter was $794 \pm 318 \mu \mathrm{m}(n=43 ; 2.5 \pm 1.0 /$ specimen $)$ in the HTARB group, $825 \pm 232 \mu \mathrm{m}(n=18 ; 3 \pm 1.9 /$ specimen $)$ in the HTCCB group, and $712 \pm 256 \mu \mathrm{m}(n=43 ; 2.3 \pm 1.1$ /specimen $)$ in the normotensive group. The percentage of the thickness of elastofibrosis to the vascular wall thickness in the HTCCB group was 81.3 $\pm 20.2 \%$. In the HTARB group, it was $39.4 \pm 13.8 \%$, and it was $39.1 \pm 11.3 \%$ in the normotensive group. The percentage of elastofibrosis in the HTCCB group was statistically higher than in the other two groups $(p<0.005, p<0.005)$. In the interlobar arteries, the elastofibrosis in the HTARB group showed no significant difference compared to that of the normotensive group.

\section{Discussion}

We reported that the long-term administration of an ARB induced remarkable morphological changes in renal afferent arteriolar walls in Zucker fatty rats in 2011 [1]. The same proliferative changes of the SMCs in the arteriolar walls were induced more frequently in young spontaneous hypertensive rats (SHRs) than adult SHRs in 2013 by the long-term administration of an another ARB [2]. We also found that the morphological changes were induced by the long-term administration of an ACEI in Zucker fatty rats [3]. However, these proliferative changes were not observed in young SHRs with the long-term administration of aliskiren, a renin inhibitor and another type of RAS blocker [7].

These days, ARBs/ACEIs are considered as first-line antihypertensive agents according to the guideline for the treatment of hypertension presented in 2014 and 2019 by the Japanese Society of Hypertension (JSH) [8, 9]. The first Japanese guideline for the treatment of hypertension by the JSH was published in 2000, and the first-line antihypertensive agents of the guideline consisted of 6 kinds of drugs (CCBs, ARBs/ACEIs, diuretics, $\beta$-blockers, $\alpha \beta$-blockers, and $\alpha$-blockers). However, the first-line antihypertensive agents were reduced in subsequent guidelines to the three kinds of CCBs, ARBs/ACEIs, and diuretics until the guideline of 2014. ARBs are very commonly used for the treatment of hypertension nowadays, because of their organ-protective effects, notably renoprotective and cardioprotective effects [4,5], although their side effects, such as hyperkalemia [10], hyponatremia [11], and acute decrease of kidney function $[12,13]$, especially in elderly people, are not infrequent.

ARBs are recommended for hypertensive patients with diabetes mellitus because of their suppression of the contraction of afferent and efferent arterioles, especially efferent, reducing glomerular hyperfiltration and transglomerular pressure by inhibiting the RAS in kidney tissue $[5,14]$. ARBs/ACEs are also recommended for their cardioprotective effects in hypertensive patients, as well as in cardiac failure and/or after myocardial infarction due to their suppressive effects in remodeling of the media in coronary arteries $[15,16]$. However, the effects of ARBs on renal arterioles and coronary arteries are quite different. In RAS, production of renin occurs in the juxtaglomerular apparatus in the kidneys and all the SMCs of afferent arteriolar walls have the potential to be renin-productive cells [17]. Therefore, RAS blockers induce a feedback reaction in RAS in kidney tissues, and consequently an increase of tissue renin production in the kidneys [18-20].

In the 1980s and 1990s, the genesis of renoprotective effects of ARBs/ACEIs were considered as introducing a dilatation of efferent arterioles much more than afferent arterioles. This reduces transglomerular pressure because greater numbers of efferent than afferent angiotensin II type 1 receptors exist [21].Therefore, ARBs/ACEIs block the contraction in the efferent arterioles more effectively than in afferent arterioles [22, 23]. In a short period, this vascular response was proven by many investigations [24]. However, our study showed remarkable proliferative changes and an irregular arrangement and size of the afferent arte- 
riolar SMCs in the HTARB group. Recently, several clinical studies that reported the treatment of ARB/ACEIs for diabetic nephropathy and/or other chronic kidney diseases showed declined kidney function over the years of long-term follow-up [25]. These altered myocytes lost the strong contractile myosin heavy chains, reducing both contraction and dilation. The morphological changes of afferent arteriolar walls were principally similar in several longterm studies which we have undertaken using different strains of rats [1-3].

In the HTCCB group, the thickening of afferent arteriolar SMCs was sometimes observed. However, the irregularities of the arrangement and/or size of SMCs were mild. These findings did not deviate from the category of abnormalities associated with hypertensive arteriolosclerosis in the kidneys, so-called benign nephrosclerosis. The number of patients in the HTCCB group was smaller than in the other two groups, but there were no hypertensive patients without RAS blockers in our hospital, because antihypertensive treatment with RAS blockers is the standard approach in Japan.

We considered that the narrowed afferent arteriolar lumens induced by ARBs could reduce the transglomerular pressure. Therefore, we suspect that these morphological changes are the main reason why RAS blockers reduce the amount of proteinuria, not only because of the dilation of efferent arterioles. These morphological changes of afferent arteriolar walls have effects for renoprotection through a decrease of the amount of blood flow into surviving glomeruli to avoid the glomerular hyperfiltration, and the decrease in intracapillary pressure in glomeruli causing initially high blood pressure. However, RAS blockers induce an overproduction of tissue renin by the feedback of RAS, especially using the high dosage of RAS blockers. In our previous studies, ARBs induced striking proliferative changes in afferent arterioles and the overproduction of tissue renin simultaneously in rat kidneys. Renin is known to activate SMCs through prorenin/renin receptors and activate intracellular proliferative systems, such as MAPKs, and these pathways are not interrupted by ARBs [26, 27]. Therefore, we suspect that the increased prorenin/renin accelerates the abnormally remarkable proliferative changes of afferent arteriolar walls.

In this study, severe proliferative changes of renal afferent arteriolar SMCs were also observed in humans. Some cases treated with a high dose of ARBs and diuretics showed striking morphological changes and extensive narrowing and meandering of the arteriolar lumens. The ratio of inner/outer diameter of arterioles positively correlated with eGFR. The results showed that the smaller values of the ratio indicated the thicker arteriolar walls, and the thicker arteriolar walls correlated with the lower glomerular filtration rate. These narrowed and meandering afferent arteriolar lumens could introduce not only the good effects for glomeruli mentioned above, but also reduce the glomerular blood flow intensity that leads to ischemia of glomeruli, which could induce tubulointerstitial damage and fibrosis.

This study was performed using nephrectomized kidneys with malignancy in the Urological Department, therefore the data on the onset of hypertension and duration of the treatment with RAS blockers were unclear. However, the morphological changes of afferent arterioles are much more frequent in patients with RAS blockers than without. We concluded that RAS blockers induce the morphological changes, narrow the afferent arteriolar lumens, and cause the disappearance of SM-2. SM-2 is one of the isoforms of myosin heavy chain, and it is the mature form of myosin with the strongest contractive force [28]. We wonder whether the high dosage and long duration of treatment, especially with RAS blockers, was really renoprotective or not. Associated with age, a decrease of eGFR will occur, and the serum levels of renoexcretive ARBs will gradually increase. In this study, the ages of the patients were limited from 45 to 74 years, meaning we need to study more elderly subjects in the future. We also consider that it is possible that the morphological changes are reversible in short-term treatment. We can measure the glomerular volume in subsequent studies [29], which might assist with the estimation of the afferent arteriolar changes and glomerular function. 


\section{Kidney \\ Blood Pressure Research}

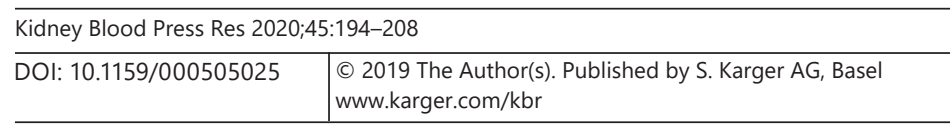

Nagai et al.: Morphological Changes of Renal Arterioles Induced by ARB Treatment

\section{Conclusions}

We studied 44 wedge resections of uninvolved parts in kidneys uninephrectomized because of malignancy. In hypertensive patients treated with antihypertensive agents, including ARBs/ACEIs, we frequently observed the marked proliferative changes of renal afferent arteriolar SMCs with irregularities in arrangement and in size. These changes were not observed in hypertensive patients without ARBs/ACEIs. We consider that these morphological changes in the renal afferent arterioles are induced by ARBs/ACEIs. We also consider that an important part of the effect of transglomerular pressure reduction is induced by these morphological changes with ARBs/ACEIs, and not according to the functional change of efferent arterioles alone. These morphological changes of renal afferent arterioles could lead to glomerular ischemia because the thicker arteriolar walls correlated with the lower glomerular filtration rate. However, the clinical consequences of these morphological changes in correlation with ARBs/ACEIs are not clear enough and require further analysis. When using ARBs/ACEIs, renal arteriolar morphological changes should be considered.

\section{Acknowledgements}

We are grateful to Toshie Shimozeki, Toho University, for her excellent technical assistance. We would also like to thank the surgeons of the Department of Urology for their co-operation, and Mitsuko Sato for her secretarial work. The authors are indebted to Prof. emeritus Chiaki Nishimura, Department of Informatics, Toho University, for his helpful suggestions during the statistical analysis.

\section{Statement of Ethics}

Our patients gave their written informed consent for inclusion and the study protocol was approved by the ethical committee on human research of Toho University Hospital.

\section{Disclosure Statement}

The authors have no conflicts of interest to declare.

\section{Funding Sources}

The research received no specific grants from any funding agency in the public, commercial, or not-for-profit sectors. Dr. Shibuya received a research grant from Sumitomo Dainippon Pharma Co. Ltd. However, he diagnosed renal cell carcinoma as one of the pathologists and was not involved in the selection of cases or the analysis of the data in this study.

\section{Author Contributions}

Research idea and study design: Y.N., F.Y., N.Y. Specimen acquisition: K.S., F.Y. Data analysis/interpretation: Y.N., Ka.N., Y.A., N.Y. Statistical analysis: Y.N., Y.S., T.I. Supervision or mentorship: Ko.N., T.M., Y.A., Y.U., N.Y. Each author contributed important intellectual content 
Nagai et al.: Morphological Changes of Renal Arterioles Induced by ARB Treatment

during the manuscript drafting or revision and accepts accountability for the overall work by ensuring that questions pertaining to the accuracy or integrity of any part of the work are appropriately investigated and resolved.

\section{References}

1 Nakanishi K, Nagai Y, Honglan Piao, Akimoto T, Kato H, Yanakieva-Georgieva N, et al. Changes in renal vessels following the long-term administration of an angiotensin II receptor blocker in Zucker fatty rats. J Renin Angiotensin Aldosterone Syst. 2011 Jun;12(2):65-74.

2 Nagai Y, Nakanishi K, Akimoto T, Yamanaka N. Proliferative changes of renal arteriolar walls induced by administration of angiotensin II receptor blocker are frequent in juvenile rats. J Renin Angiotensin Aldosterone Syst. 2014 Dec;15(4):440-9.

3 Nakanishi K, Nagai Y, Akimoto T, Yamanaka N. Changes in renal vessels associated with long-term administration of angiotensin converting enzyme inhibitor in Zucker fatty rats. J Smooth Muscle Res. 2017;53(0): 20-30.

4 Düsing R: Mega clinical trials which have shaped the RAS intervention clinical practice. Ther Adv Cardiovasc Dis. 2016;10:(3):133-50.

5 Kobori H, Mori H, Masaki T, Nishiyama A. Angiotensin II blockade and renal protection. Curr Pharm Des. 2013; 19(17):3033-42.

6 Ouchi Y, Rakugi H, Arai H, Akishita M, Ito H, Toba K, et al.; Joint Committee of Japan Gerontological Society (JGLS) and Japan Geriatrics Society (JGS) on the definition and classification of the elderly. Redefining the elderly as aged 75 years and older: Proposal from the Joint Committee of Japan Gerontological Society and the Japan Geriatrics Society. Geriatr Gerontol Int. 2017 Jul;17(7):1045-7.

7 Nagai Y, Nakanishi K, Yamanaka N. Direct renin inhibitor is better than angiotensin II blocker for intrarenal arterioles. Kidney Blood Press Res. 2016;41(5):561-9.

8 Japanese Society of Hypertension. Guidelines for the Management of Hypertension 2014. Tokyo, Japan: Life Science Publishing Co. Ltd.; 2014.

9 Japanese Society of Hypertension. Guidelines for the Management of 2 Hypertension 2019. Tokyo, Japan: Life Science Publishing Co. Ltd.; 2019.

10 Saito Y, Yamamoto H, Nakajima H, Takahashi O, Komatsu Y. Incidence of and risk factors for newly diagnosed hyperkalemia after hospital discharge in non-dialysis-dependent CKD patients treated with RAS inhibitors. PLoS One. 2017 Sep;12(9):e0184402.

11 Yamada H, Asano T, Aoki A, Ikoma A, Yoshida M, Kusaka I, et al. Combination therapy of angiotensin II receptor blocker and thiazide produces severe hyponatremia in elderly hypertensive subjects. Intern Med. 2014;53(7): 749-52.

12 Mansfield KE, Nitsch D, Smeeth L, Bhaskaran K, Tomlinson LA. Prescription of renin-angiotensin system blockers and risk of acute kidney injury: a population-based cohort study. BMJ Open. 2016 Dec;6(12):e012690.

13 Turgutalp K, Bardak S, Horoz M, Helvacı I, Demir S, Kiykim AA. Clinical outcomes of acute kidney injury developing outside the hospital in elderly. Int Urol Nephrol. 2017 Jan;49(1):113-21.

14 St Peter WL, Odum LE, Whaley-Connell AT. To RAS or not to RAS? The evidence for and cautions with reninangiotensin system inhibition in patients with diabetic kidney disease. Pharmacotherapy. 2013 May; 33(5): 496-514.

15 Akazawa H, Yabumoto C, Yano M, Kudo-Sakamoto Y, Komuro I. ARB and cardioprotection. Cardiovasc Drugs Ther. 2013 Apr;27(2):155-60.

16 Lemesle G, Lamblin N, Meurice T, Tricot O, Bauters C. Angiotensin II receptor blockers versus angiotensinconverting enzyme inhibitors in patients with stable coronary artery disease: Prevalence, correlates, and prognostic impact (from the CORONOR study). J Cardiol. 2017 Mar;69(3):542-7.

17 Bonsib SM. Renal anatomy and histology. In: Janette JC, Silva F, Olson JL, D'Agati VD, editors. Heptinstall's pathology of the kidney. 7th ed. Philadelphia (PA): Wolters Kluwer; 2015. pp. 1-66.

18 Owen RA, Molon-Noblot S, Hubert MF, Kindt MV, Keenan KP, Eydelloth RS. The morphology of juxtaglomerular cell hyperplasia and hypertrophy in normotensive rats and monkeys given an angiotensin II receptor antagonist. Toxicol Pathol. 1994 Nov-Dec;22(6):606-19.

19 Jackson DG, Jones HB. Histopathological and ultrastructural changes in the juxtaglomerular apparatus of the rat following administration of ZENECA ZD6888 (2-ethyl-5,6,7,8-tetrahydro-4-[(2'-(1H-tetrazol-5-yl) biphenyl-4-yl)- methoxy]quinoline), an angiotensin II antagonist. Toxicol Pathol. 1995 Jan-Feb;23(1):7-15.

20 Doughty SE, Ferrier RK, Hillan KJ, Jackson DG. The effects of ZENECA ZD8731, an angiotensin II antagonist, on renin expression by juxtaglomerular cells in the rat: comparison of protein and mRNA expression as detected by immunohistochemistry and in situ hybridization. Toxicol Pathol. 1995 May-Jun;23(3):256-61.

21 Razga Z, Nyengaard JR. Up- and down-regulation of angiotensin II AT1-A and AT1-B receptors in afferent and efferent rat kidney arterioles. J Renin Angiotensin Aldosterone Syst. 2008 Dec;9(4):196-201.

22 Pelayo JC, Quan AH, Shanley PF. Angiotensin II control of the renal microcirculation in rats with reduced renal mass. Am J Physiol. 1990 Feb;258(2 Pt 2):F414-22. 
23 Blantz RC, Gabbai FB. Glomerular hemodynamics in pathophysiologic conditions. Am J Hypertens. 1989 Nov; 2(11 Pt 2):208S-12S.

24 Kobori H, Nangaku M, Navar LG, Nishiyama A. The intrarenal renin-angiotensin system: from physiology to the pathobiology of hypertension and kidney disease. Pharmacol Rev. 2007 Sep;59(3):251-87.

25 Komaroff M, Tedla F, Helzner E, Joseph MA. Antihypertensive medication and change in stages of chronic kidney disease. Int J Chronic Dis. 2018 Feb;2018:1382705.

26 Liu G, Hitomi H, Hosomi N, Shibayama Y, Nakano D, Kiyomoto H, et al. Prorenin induces vascular smooth muscle cell proliferation and hypertrophy via epidermal growth factor receptor-mediated extracellular signal-regulated kinase and Akt activation pathway. J Hypertens. 2011 Apr;29(4):696-705.

27 Nguyen G, Delarue F, Burcklé C, Bouzhir L, Giller T, Sraer JD. Pivotal role of the renin/prorenin receptor in angiotensin II production and cellular responses to renin. J Clin Invest. 2002 Jun;109(11):1417-27.

28 Kim HS, Aikawa M, Kimura K, Kuro-o M, Nakahara K, Suzuki T, et al. Ductus arteriosus. Advanced differentiation of smooth muscle cells demonstrated by myosin heavy chain isoform expression in rabbits. Circulation. 1993 Oct;88(4 Pt 1):1804-10.

29 Viggiano D, Nigro M, Sessa F, Vignolini G, Campi R, Serni S, et al. The number of nephrons in different glomerular diseases. PeerJ. 2019;7:e7460. 